\title{
Agresión, victimización y armas en los centros educativos de San Salvador2
}

\section{Introducción}

Nadie duda que, en la actualidad, la violencia en El Salvador es un problema grave. Después del fin de la guerra y con la firma del tratado de paz, las encueslas de opinión pública comenzaron a acusar a la delincuencia y la violencia como unos de los principales problemas del país; la preocupación por el problema de la violencia llegó a tener tal magnitud que, en los últimos tres años, los ciudadanos han estado más alarmados por este problema que por la guerra civil a mediados de la década pasada (Cruz y González, 1997). En buena medida esa preocupación tiene un referente objetivo en la realidad, El Salvador figura como uno de los países con las tasas de homicidios más elevadas del continente (Iglesias, 1997). De acuerdo con la Fiscalía General de la República, en 1995 murieron un poco más de 7,000 personas por violencia intencional; para 1997 , los registros indican que el total de personas muertas por causa de asesinato es mayor de 5,000.

Más allá de la estéril discusión de si El Salvador es el país más violento o no de Latinoamérica o de la región, los datos anteriores y los ofrecidos por los estudios locales y los organismos internacionales imponen la innegable realidad de que, con esos niveles de violencia social, no puede existir un desarrollo sostenible. Efectivamente, con magnitudes tales, la violencia no sólo afecta la integridad física y psicológica de muchos ciudadanos, esto es, la salud; lambién afecta los procesos de interrelación social y vulneran la capacidad de un país para destinar la mayor parte de sus energías produclivas para el desarrollo. Lo que en otras circunstancias se destinaría para mejorar las condiciones de vida, de suyo precarias, de la mayor parte de la población y para invertir en el desarrollo social, debe desviarse para atender las secuelas de la violencia, para protegerse de la misma y cubrir las pérdidas ocasionadas por la desaparición forzada e inesperada de los agentes de producción, esto es, de la misma población econó- 
micamente activa. Esto tiene un impacto aún mayor cuando el tipo de población afectada por la violencia es la que posee el mayor potencial de desarrollo de cara al futuro.

Y es que la violencia, ésa que se ha hecho tan cotidiana en los últimos años en toda la región latinoamericana, afecta a todos los grupos sociales pero, en especial, a los jóvenes, esto es, a las personas entre 13 y 25 años de edad. En este punto no parecen haber divergencias en los estudios multicénıricos y en las encuestas de viclimización: la mayor parte, por no decir todos, señalan que el grupo más afectado por la violencia lo constituyen los jóvenes, especialmente los hombres (Cruz, 1998; U.S. Department of Justice, 1997). La mayoría de las sociedades contemporáneas han visto cómo, en los últimas décadas, la juventud se ha convertido en uno de los sectores poblacionales más afectados por la violencia al constituirse en las víclimas y los victimarios más frecuentes de las agresiones (Levine y Rosich, 1996). En Estados Unidos, en donde se ha estudiado con más insistencia el perfil demográfico de los aclores que participan en el fenómeno de la violencia, se ha llegado a concluir que la edad constiluye "uno de los más importantes predictores singulares del riesgo de victimización por violencia" (National Research Council, 1993, p. 68). En la mayor parte de países de Latinoamérica estudiados, Yunes y Rajs encontraron que las tasas de mortalidad por causas violentas es mucho mayor entre los adolescentes y jóvenes que en cualquier otro grupo de edad (1994). En estas lendencias, El Salvador no es la excepción. Basado en los expedientes del Instituto de Medicina Legal, Cruz (1997) encontró que la mayor parte de homicidios son cometidos en contra de jóvenes del sexo masculino cuyas edades oscilan entre los 16 y 25 años de edad; más aún, según los cálculos realizados con tales expedientes, un joven de 17 años tiene cuatro veces más probabilidades de ser asesinado en $\mathrm{El}$ Salvador que un hombre de 50 años.

Pero no sólo la edad parece ser un "prediclor" importante, las investigaciones demográficas scñalan también al sexo como una variable fundamental en la incidencia de la violencia: los hombres aparecen como las víctimas más frecuentes de la violencia que lleva a la muerte, esto es, la violencia homicida. Otra revisión de las tasas de muertes violentas de los años ochenta reveló que en todos los países estudiados de América Latina y el Caribe, la relación entre las tasas masculina y femenina fue siempre mayor de 1 y en todos - a excepción de Cuba - la diferencia fue sustancial (Yunes, 1993). De tal forma que, con sus variantes en cada uno de los paises, las personas más expuestas a la violencia letal son los hombres jóvenes, $y$ en la medida en que una sociedad tiene mayores problemas de violencia, en esa medida sus jóvenes tienen mayor probabilidad de morir por un incidente violento.

Dados los niveles peculiares de violencia que existen en El Salvador, el impacto de la misma en la juventud es decisivo. Las consecuencias de la violen- 
cia están condicionadas por las expresiones del fenómeno en el caso salvadoreño. El creciente fenómeno de las pandillas o "maras" callejeras es una de las expresiones de esa violencia. De acuerdo con informaciones provenientes de la Policía Nacional Civil, en el Área Metropolitana de San Salvador subsisten alrededor de 20,000 organizados en pandillas y, a juzgar por la presencia de este lipo de grupos en todo el país, es probable que el número nacional llegue o sea más del doble. No hay duda de que este fenómeno de pandillas callejeras, con sus expresiones que recuerdan a las "gangs" de Los Angeles, constituye una fuente importante de violencia y de victimización entre la juventud (Smut y Miranda, 1997), pero no es la única.

El problema de las riñas o batallas campales en plena vía pública protagonizada por estudiantes de distintos centros educativos constituye otra importante manifestación de violencia, aunque quizás con menos letalidad que la prolagonizada por las maras callejeras. En los últimos años, la población urbana ha sido testigo de los constantes enlrentamienlos en el centro de San Salvador, protagonizados por grupos pertenecientes a centros educativos; en ocasiones, tales confrontaciones ponen en peligro la seguridad de los transeúntes o de los vecinos donde los hechos ocurren dada la virulencia con que se desarrollan tales incidentes (Martínez, 1998). Estudios han mostrado la conexión de estos grupos, conocidos también como "maras" y con el adjelivo de "estudiantiles", con el desartollo de las condiciones para que los jóvenes se integren a las maras o pandillas callejeras; aunque los fenómenos son distintos por su dinámica y expresión, tales estudios han sugerido que la pertenencia a maras estudiantiles - salvando las particularidades - podría ser una elapa previa para la inlegración a las maras callejeras (Cruz y Portillo, 1998).

Lo anterior muestra la importancia de los comportamientos violentos en el contexto escolar y pone de manifiesto el hecho de que la escuela sea uno de los ambientes en los que la violencia se concreta cotidianamente. Estudios en el exterior han mostrado la vinculación entre la violencia que se genera en los centros de educación media y los niveles de criminalidad que reportan las comunidades en las que se encuentran tales instituciones escolares (Toby, 1994; citado por Levine y Rosich, 1996). Otras investigaciones han revelado que el ambiente escolar es una variable importante para definir la aparición de lo que algunos investigadores llaman "la aparición del riesgo de delincuencia"" (Tolan, 1997). Mientras que otros estudios revelan que, aunque el fenómeno de las pandillas no se origina en las escuelas, éstas se convierten en el terreno adecuado para el reclutamiento de nuevos pandilleros $y$, en ocasiones, para la planeación de hechos de violencia en contra de otros grupos (Office of Juvenile Justice and Delincuency Prevention, 1994). Y es que las conductas de agresión dentro de los centros escolares son también una expresión de la violencia que no puede ser soslayada. Investigaciones realizadas en otros países, en especial Estados Unidos, revelan que esta problemática se ha venido incrementando desde la década 
de los años ochenta, de tal forma que el riesgo de sufrir alguna agresión dentro del centro escolar o por compañeros estudiantes es ahora más alto entre los jóvenes entre 12 y 17 años en Estados Unidos que en e! pasado (Lockwood, 1997).

En El Salvador no se tiene una medida sobre ese problema; sin embargo, a juzgar por las noticias de la prensa, pareciera que la violencia asociada a la población estudiantil es ahora más grave que antes. La prensa nacional reportó entre 1996 y 1997 más de 600 heridos y decenas de muertos a causa de las riñas estudiantiles en las calles de la capital salvadoreña (Urías, 1997). Y es que mucho se ha hablado de la magnitud de esas problemáticas, pero es muy poco lo que se ha investigado sobre las expresiones y la dinámica de las mismas. Como ya se ha dicho anteriomente, los jóvenes no sólo resultan ser las víctimas más frecuentes, sino que se están convirtiendo también, con elevada frecuencia, en los perpetradores más comunes de la violencia.

Por ello, como parte de un esfuerzo muy amplio para estudiar la violencia en El Salvador, a finales de 1996, el Instituto Universitario de Opinión Pública de la UCA, con el apoyo financiero de Rädda Bamen, desarrolló un estudio de campo para recoger las conductas y actitudes de los adolescentes en instituciones educativas acerca de la violencia en el Area Metropolitana de San Salvador (AMSS). El estudio se basa en un instrumento que fue desarrollado originalmente por psicólogos de la Escuela de Salud Pública de la Universidad de HoustonTexas, el cual ha sido aplicado en otras tres ciudades: Houston, Iowa y Salvador de Bahía, Brasil. El presente artículo es un breve avance de algunos de los resultados de ese estudio, el cual abarca una serie de variables relacionadas con la violencia dentro de las escuelas, pero del cual para esta entrega se utilizarán esencialmente los que se refieren a tres variables: $(a)$ la frecuencia de comportamientos agresivos; (b) la frecuencia de la victimización por tales comportamientos, y (c) la conducta de acarreo de armas por parte de los jóvenes en el interior de los centros educativos. Este artículo tiene, por tanto, fines descriptivos: pretende ofrecer la información referida a esos comportamientos siguiendo el esquema tradicional de presentar la metodología del estudio, mostrar los resultados y hacer algunas reflexiones sobre los mismos. En cualquier forma, es el primer paso del estudio de los factores que están asociados a la violencia estudiantil en el Area Metropolitana de San Salvador.

\section{Aspectos metodológicos}

\subsection{Muestra de la población estudiada}

La investigación fue realizada a un tolal de 1,032 alumnos de educación básica y media (de séptimo grado a tercer año de bachillerato), que constituían una muestra representativa de la población estudiantil del AMSS y cuyas edades 
oscilaban entre los 13 y 24 años de edad. El 55.5 por ciento de los jóvenes estudiados pertenecen al sexo masculino, mientras que el $\mathbf{4 4 . 5}$ por ciento pertenece al sexo femenino. La mayor parte de los jóvenes encuestados tenían entre 16 y 18 años de edad - en el momento en que se llevó a cabo el trabajo de campoy la edad promedio de toda la muestra era de 16.2 años, con una desviación típica de 1.9 años. El 38.8 por ciento de los consultados estaba estudiando entre séptimo y noveno grado; mientras que el 61.2 por ciento se encontraba en bachillerato. Al igual que los adultos, el 19 por ciento de los jóvenes abordados declaró no tener confesión religiosa alguna; el 56 por ciento se declaró católico -entre practicante y no practicante- y el 22.5 por ciento se reafirmó como miembro de otras religiones, en especial la "cristiana-evangélica". Finalmente, el 87 por ciento de los jóvenes declararon que no tenían empleo en el momento del estudio, mientras que el resto sí se encontraba trabajando.

\subsection{El instrumento}

El cuestionario utilizado para estudiar el problema de la violencia en los jóvenes de los centros educativos, es una adaptación al español de uno desarrollado por miembros de la Escuela de Salud Pública de la Universidad de HouslonTexas, que incorpora una serie de escalas para medir la violencia en las escuelas de educación media (Orpinas, 1997), más algunos reactivos incorporados localmente. En total, el cuestionario contenía 95 ítems, la mayoría de los cuales formaban escalas y buscaban medir los siguientes aspectos de comportamiento y actilud: la Escala de agresión (diseñada por Orpinas y Frankowski, 1996), peleas en la escuela, portación de amas, exposición a la violencia en la comunidad, actitudes hacia la violencia, habilidades para resolver conflictos, conductas de consumo excesivo de alcohol y drogas, consejería sobre violencia de los padres, nivel de comunicación y empatía con los padres y expectativas sobre el futuro. El instrumento contenía también reaclivos para conocer las características demográficas de los jóvenes: sexo, edad, nivel educativo, condición laboral, confesión religiosa, municipio de residencia, entre otras cosas. En esta breve presentación de los resultados se hará uso esencialmente de tres escalas, más algunas de las variables demográficas. Las escalas referidas son: Escala de agresión, compuesta por 11 reactivos; cada uno tenía siete posibles respuestas e indicaban la frecuencia de la conducta de los jóvenes, en el lapso de la semana, antes de que se realizara la encuesta. Esta escala obtuvo una consistencia interna muy alta (Alfa de Cronbach $=0.80$ ). Los ílerns de la escala de agresión son los siguientes: yo hice bromas (molesté) a otros estudiantes para que se enojaran; yo me enojé fácilmente con otra persona; yo respondl con golpes cuando alguien me golpé primero; yo dije cosas sobre otra persona para hacer reir a los estudiantes; yo alenté (estimulé, aconsejé) a otros estudiantes para pelear; yo empujé a otros estudiantes; yo estuve enojado la mayor parte del dia; yo peleé a golpes (pelear o puños, tirar el pelo, morder) porque estaba enojado; yo le di una bofetada 
(cachetada, palmada) o patada a alguien; yo insulté a otros estudiantes (les dije malas palabras) y yo amenacé a alguien con herirlo o pegarle.

El esludio de los comporlamientos relacionados con la violencia en los centros educalivos incluyó también una batería de ítems que conformaban una escala de victimización. Esta escala recogía la frecuencia de comportamientos agresivos sufridos por los estudiantes en la última semana lectiva y exhibió una confiabilidad estadística también elevada (Cronbach $=0.776$ ). Los ítems contenidos en esta escala son: un estudiante me hizo bromas (me molestó) para que yo me enojara; un estudiante me dio una golpiza; un estudiante dijo cosas sobre mi para hacer reir a otros estudiantes; otros estudiantes me alentaron (estimularon, aconsejaron) para pelear; un es(udiante me empuj6; un estudiante me invitó a pelear; un estudiante me dio una bofetada (cachetada, palmada) o patada; un estudiante me insultó a mr o a mi familia; un estudiante me amenazó con herirme o golpearme y un estudiante trató de herir mis sentimientos.

Finalmente, en este trabajo se expondrán los resultados referidos a la escala de portación de armas dentro de la escuela. Esta escala contenía cuatro reactivos que medían la frecuencia de días en que el joven acudió al centro educativo portando algún arma (pistola o revólver, rifle, armas largas de fuego, cuchillo o navajas, palos o cachiporras). El coeficiente de confiabilidad fue de 0.92 .

\section{Procedimiento}

La muestra de los jóvenes se seleccionó con base en los datos de distribución escolar pública y privada sobre los centros educalivos del AMSS, proporcionados por la oficina de estadística del Ministerio de Educación. La definición de la muestra se hizo siguiendo un proceso multielápico. En primer lugar se seleccionaron 34 centros educativos de educación media por medio de una estratificación por tarnaño y tipo de institución (pública y privada), y dentro de cada estrato se hizo una selección aleatoria de los centros. En segundo lugar, en cada una de las instiluciones se seleccionaron aleatoriamente los grados, cursos o secciones entre séptimo grado y tercer año de bachillerato que serían encuestados; finalmente, los jóvenes eran seleccionados en cada curso mediante un sorteo: los "favorecidos" debían quedarse y llenar por su cuenta el cuestionario bajo la supervisión de dos "aplicadores", el resto de jóvenes (los no seleccionados y quienes no cumplían con los requisitos de edad) debían abandonar el salón. Cada cuestionario se contestó individualmente por los estudiantes, mientras uno de los encuestadores leía las preguntas y otro se encargaba de atender las dudas. Todos los cuestionarios fueron codificados y procesados por computadora usando el programa SPSS (Norusis, 1991).

Para comprobar la asociación entre las variables usadas en este trabajo se usaron las pruebas estadísticas de Análisis de la varianza (ANOVA), Eta y ChiCuadrada, dependiendo de las características de las variables conjugadas. 


\section{Resultados}

\subsection{Las conductas de pelea}

Para comenzar, los resultados muestran que cerca del 20 por ciento de los estudiantes consultados admitieron haber peleado dentro de la escuela en los últimos 30 días; mientras que del resto, el 76.3 por ciento afimó no haber peleado ninguna vez y el 4.4 por ciento se abstuvo de responder al reactivo4. Este resultado es mucho más elevado que el reportado por el sistema educativo estadounidense, el cual fue del 16 por ciento para el lapso de un año (Lockwood, 1997). La mayor parte de los jóvenes que declararon haber tenido un enfrentamiento (pelear a golpes, jalar el pelo, morder, etc.), afimaron que pelearon en una ocasión en el transcurso del mes, mientras que el resto lo hizo en dos ocasiones o más. Por olro lado, a los jóvenes se les preguntó también sobre el número de veces que, en el último mes, participaron en una pelea -adentro o afuera de la escuela $-y$ de la cual hayan resultado con golpes o heridas que hayan ameritado atención médicas; esto implicaba un incidente de mayor intensidad que el recogido por el ílem anterior. El 85.5 por ciento de los jóvenes contestó que no había pasado por una situación de esa naturaleza, mientras que el 8.4 por ciento dijo que sí había pasado por eso, al menos en una ocasión en los últimos 30 días; el reslo (5.8 por ciento) se absluvo de responder a la pregunta. En otras palabras, lo anterior quiere decir que al menos ocho de cada cien estudiantes de secundaria, entre 13 y 19 años, participaron en un hecho violento del cual salieron sensiblemente lastimados.

Una primera comparación entre ambos reactivos mostró un importante nivel de correlación entre las conductas de pelea dentro de la escuela y las conductas de enfrentamiento en las cuales se salió herido o golpeado (Pearson $=0.30094$, $p<0.0001$ ). Esto sugiere que ambas conductas son paralelas en los jóvenes estudiantes del Area Metropolitana de San Salvador: en la medida en que los estudiantes protagonizan peleas dentro del centro educativo, es más probable que se vean involucrados también en eventos de agresión en cualquier otro entomo y del cual pueden salir heridos. Y no sólo eso. Estos datos ofrecen evidencias de que los jóvenes que protagonizan peleas o que tienen problemas de conducla agresiva dentro de los centros educativos serían los más propensos a sufrir algún percance por participar en las riñas o enfrentamientos callejeros.

\subsection{Las conductas de agresión dentro de la escuela}

Ahora bien, los reactivos anteriores mostraron también un elevado significativo nivel de correlación con la Escala de agresión utilizada en el cuestionario. El ítem de peleas dentro de la escuela tuvo un coeficiente de correlación con la escala de agresión de 0.3704 ( $p<0.0001$ ), validando en cierta forma dicha escala. Esta escala de agresión medía la frecuencia de las conductas agresivas entre los 
estudiantes en unos valores que iban desde cero (0), indicando la ausencia de conductas agresivas en el transcurso de una semana, hasta sesenta y seis (66), como valor de la mayor cantidad de conductas agresivas en el mismo lapso de tiempo. El valor promedio (índice) que resultó de la escala con las respuestas de los 1,034 jóvenes fue de 9.81, con desviación típica de 9.62. Cada punto, en este caso, representa una conducta agresiva cometida en la semana anterior a la encuesta. Esto significa que, aproximadamente, los jóvenes del AMSS tuvieron una media de diez comportamientos agresivos en el transcurso de la semana anterior a la aplicación del instrumento.

Sin embargo, los índices de conductas de agresión no son iguales para todos los jóvenes. Los resultados de la escala según distintas variables, expuestos en el Cuadro 1, muestran que es posible encontrar diferencias en el comportamiento de los jóvenes de acuerdo con ciertas variables. Por ejemplo, es notoria la diferencia en el índice de los hombres y las mujeres: los adolescentes del sexo masculino presentan prácticamente el doble de conductas agresivas que sus compañeras del sexo femenino, esta diferencia tiene una elevada significación estadística $(p<0.0001)$, y parece ser la variable de más peso asociativo con la escala de agresión. En el caso de la edad, las diferencias en los índices son menos intensas, pero no por ello dejan de ser significativas o interesantes. En este caso, los datos sugieren que a medida que los jóvenes tienen más edad, las expresiones de agresión dentro de los cenlros escolares del Area Metropolitana de San Salvador se reducen $(p<0.05)$. Esto constiluye una tendencia muy particular, sobre todo porque, al ver las tendencias de involucramiento de los jóvenes en hechos de violencia en riñas callejeras, se esperaría que con el aumento de la edad existiera lambién un aumento en las conductas agresivas en el interior de los centros escolares. Los datos más bien rechazan esa suposición, e indican que los más jóvenes están más propensos a agredir a sus compañeros al menos dentro del contexto escolar. Otra variable que parece fundamental en la asociación con la agresividad de los jóvenes dentro del contexto escolar es la religión. De acuerdo con los promedios expuestos en el Cuadro 1, los muchachos que presentan el mayor índice de conductas agresivas en el transcurso de un mes son quienes dicen no poseer una confesión religiosa determinada y los que pertenecen a la fe católica; por el contrario, los llamados "cristianos-evangélicos", es decir, los seguidores de iglesias protestantes pentecostales y los que forman parte de otras confesiones religiosas (Testigos de Jehová, Mormones, Fe Bahai', etc.) exhiben un índice particularmente bajo y los hace diferenciarse claramente de los católicos y no religiosos $(p<0.001)^{6}$. Lo anterior significa que la pertenencia a una religión concreta puede hacer una diferencia en el comportamiento agresivo de los jóvenes; según los dalos, las religiones "no oficiales" $\longrightarrow$ al menos las no tradicionales - tendrían más éxito en evitar que sus jóvenes afiliados sean agresivos dentro de los entornos escolares. 
Por el otro lado, las variables que no parecen tener una relación con los índices de agresión son el nivel educativo del entrevistado y el tipo de institución en la que estudian los jóvenes, definida ésta como pública o privada según el tipo de administración. Aunque es posible ver una diferencia en los índices de agresión entre los jóvenes de tercer ciclo y bachillerato, y entre quienes estudian en centros públicos de aquéllos que lo hacen en centros privados, los valores de $\mathrm{F}$ son muy bajos, de tal manera que la variabilidad no llega a ser estadísticamente significativa. De hecho, es probable que las leves diferencias entre los índices del nivel educativo se deban más a un efecto indirecto de la edad de los jóvenes que al nivel de educación en sí mismo. En el caso de las instituciones, lo que muestran los datos es que no hay diferencias entre las instituciones si éstas son clasificadas como públicas o privadas, aunque puede ser posible encontrar diferencias en las mismas si éstas son clasificadas en función de otros criterios (como tamaño de la institución, estrato socioeconómico de la población que atiende, etc.).

\section{Cuadro 1}

Indices de agresión y victimización en la escuela según variables y valores de $\mathbf{F}$

\begin{tabular}{|c|c|c|}
\hline $\begin{array}{l}\text { Indice de } \\
\text { variables }\end{array}$ & $\begin{array}{l}\text { Indice de } \\
\text { agresión }\end{array}$ & Victimización \\
\hline Todos & 9.81 & 7.26 \\
\hline $\begin{array}{l}\text { Sexo } \\
\text { Masculino } \\
\text { Femenino } \\
F\end{array}$ & $\begin{array}{c}12.31 \\
6.68 \\
95.08\end{array}$ & $\begin{array}{l}5.36 \\
8.78 \\
54.7\end{array}$ \\
\hline $\begin{array}{l}\text { Edad } \\
\text { De } 13 \text { a } 14 \text { años } \\
\text { De } 15 \text { a } 17 \text { años } \\
18 \text { años y más } \\
F\end{array}$ & $\begin{array}{r}10.79 \\
10.06 \\
8.43 \\
3.93\end{array}$ & $\begin{array}{l}7.00 \\
7.51 \\
6.90 \\
0.73\end{array}$ \\
\hline $\begin{array}{l}\text { Nivel educativo } \\
\text { Tercer ciclo } \\
\text { Bachillerato } \\
F\end{array}$ & $\begin{array}{c}10.15 \\
9.59 \\
0.82\end{array}$ & $\begin{array}{l}7.15 \\
7.32 \\
0.11\end{array}$ \\
\hline $\begin{array}{l}\text { Religión } \\
\text { Ninguna } \\
\text { Católica } \\
\text { Evangélica } \\
\text { Otras } \\
F\end{array}$ & $\begin{array}{r}10.51 \\
10.50 \\
7.67 \\
6.25 \\
7.04^{\star}\end{array}$ & $\begin{array}{l}7.87 \\
7.43 \\
6.27 \\
5.48 \\
2.62\end{array}$ \\
\hline $\begin{array}{l}\text { Tipo de instifución } \\
\text { Pública } \\
\text { Privada } \\
\text { F }\end{array}$ & $\begin{array}{c}9.33 \\
10.02 \\
1.13\end{array}$ & $\begin{array}{l}7.52 \\
7.14 \\
0.54\end{array}$ \\
\hline
\end{tabular}




\section{La victimización por conductas de agresión}

La escala de victimización mide la frecuencia de conductas de agresión que ha sufrido el joven estudiante en el lapso del último mes. Constituye el lado opuesto de la escala de agresión pues, como su nombre lo indica, se refiere al nivel de viclimización sufrido dentro de la escuela. Sin embargo, ambas escalas parecen ser muy cercanas en la práclica. El primer dato sobresaliente de la escala de victimización usada en el cuestionario que el IUDOP utilizó para medir las conduclas de violencia dentro de los centros educativos es el que se refiere a la correlación de ésta con la escala de agresión. De acuerdo con los resultados, el coeficiente de correlación de Pearson entre ambas escalas es de 0.579 ( $p<0.0001$ ), lo que indica una asociación entre el número de comportamientos agresivos en la escuela y el número de veces que ha sufrido por un comportamiento. En otras palabras, en la medida en que un joven tiende a ser más agresivo con sus compañeros de clase o de institución, en esa medida parece estar más propenso o expuesto a sufrir de alguna conducta de agresión dentro de la institución. Pero esta escala no sólo se correlaciona con la escala de agresión, sino también con los ítems de conductas de violencia que terminan en lesiones. Aunque el coeficiente de correlación no es muy alto (Pearson $=0.191$ ), sí llega a ser estadísticamente significativo, lo que indicaría que la viclimización dentro de la escuela está, de alguna manera, relacionada con los comportamientos de violencia del estudiante.

Pero más que esas asociaciones simples entre variables, todos estos datos estarían planteando el fenómeno de que los jóvenes que se comportan de manera agresiva son los mismos que sufren con más frecuencia por el mismo tipo de comportamiento, inclusive en contextos de mayor riesgo. Esto concuerda con los hallazgos de otras investigaciones que señalan a los victimarios como las víctimas más frecuentes de la violencia, sobre todo, entre el grupo de 15 y 25 años de edad (National Resarch Council, 1993).

Pero, en todo caso, ¿cuáles son las variables personales o contextuales que aparecen más asociadas a la victimización por conductas de agresión dentro de los centros escolares? De nuevo se halla al sexo y a la religión, pero la edad, el nivel escolar y el tipo de asociación no parecen estar asociadas, al menos con significancia estadística $(p<0.05)$. El promedio de conductas agresivas sufridas por un joven escolar del Area Metropolitana de San Salvador en el transcurso de una semana es de 7.2, es decir, ha enfrentado como media un total de siele comportamientos de agresión. Los jóvenes del sexo masculino son los que enfrentan tales comportamientos de agresión más que las mujeres; sin embargo, la diferencia en la victimización respecto a las mujeres no parece ser tan amplia como en ei caso de las agresiones. La otra variable importante asociada a la victimización es la confesión religiosa. Al igual que con las conductas de agresión, los jóvenes que no tienen religión y los católicos -pero especialmente los 
primeros— son los que sufren por la conducta de otros con más frecuencia. Esta asociación parece confirmar la idea de que mientras más se involucren los adolescentes en eventos violentos, con más frecuencia van a salir perjudicados.

Lo que es interesante, por otro lado, es el hecho de que la edad, con los rangos de los que se dispuso en la encuesta (de 13 a 19 años), no parece estar asociada a la victimización por comportamientos agresivos dentro de la escuela; esto a pesar de que los resultados muestran diferencias importantes en las conductas de agresión según edad, sobre todo en los grupos más jóvenes. La explicación a eslo podría encontrarse en que los comportamientos de agresión más frecuentes en las primeras etapas de la adolescencia se vuelven menos frecuentes hacia un joven que tiene más edad y, por consiguiente, más capacidad de defenderse de los ataques. En todo caso, en términos de hipótesis, puede que esas nuevas polencialidades de comportamiento lo hagan más vulnerable en el exterior de la escuela, en sus relaciones con adultos y con la dinámica de las calles y no en el interior de los centros escolares.

\subsection{La portación de armas dentro de los centros escolares}

La investigación realizada por el IUDOP aportó datos muy importantes sobre el hábito de portar armas dentro de los centros educativos por parte de los estudiantes entre 13 y 19 años del Area Metropolitana de San Salvador. La batería destinada para recoger tales hábitos reportó la frecuencia de veces que, en el lapso de un mes, los esludiantes han acudido a la escuela llevando cualquier tipo de armas (pistolas, explosivos, ammas blancas y garrotes o palos). De acuerdo con los resultados generales, que se exponen en la Gráfica 1 , un poco más del 18 por ciento de los estudiantes consultados admitieron haber traído a su escuela, colegio o centro de estudio, algún tipo de amma antes señalada y eso en el lapso de un mes. Las armas blancas aparecen como las más comunes, con un 14.4 por ciento de estudiantes que reconocieron portar alguna dentro de la escuela, le sigue la portación de garrotes, palos o porras con el 7.4 por ciento y luego vienen las armas de fuego: el 4.5 por ciento anduvo con una pistola o ama corta y el 2.5 por ciento introdujo consigo una arma larga o explosivo (como granada).

Estos porcentajes son significalivos para el tipo de conducta de la cual se está hablando. Aunque parezca baja la proporción de jóvenes que traen un explosivo o arma de fuego a la escuela, contextualizando, los datos anteriores revelan que - como promedio- de cada cien estudiantes que hay en San Salvador, al menos dos llevan consigo un explosivo dentro de su institución y catorce están armados con un cuchillo o navaja. Eso obviamente tiene repercusiones en la incidencia de la violencia generada por el comportamiento de estos jóvenes.

Pero, ¿qué variables aparecen asociadas a la portación de ammas dentro de la escuela por parte de los estudiantes? De acuerdo con los resultados generales, el sexo, el nivel educativo, la confesión religiosa y la condición de empleo son las 


\section{Gráfica 1 \\ Jóvenes que han asistido armados a la escuela \\ en el lapso de un mes según tipo de arma}

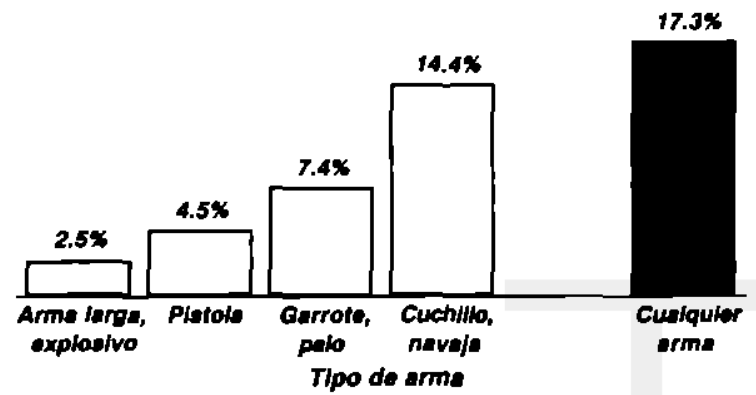

variables que aparecen asociadas a los estudiantes que acuden a los centros de estudio portando armas de cualquier tipo con mayor frecuencia (ver Cuadro 2). Los jóvenes del sexo masculino - siguiendo un comportamiento inexorable - reportaron la portación de ammas en un porcentaje más de dos veces mayor que el consignado por sus compañeras del sexo opuesto. Por su parte, los estudiantes de los niveles de bachillerato, especialmente los que se encontraban cursando el segundo año de bachillerato en 1996, reportaron un porcentaje levemente mayor - pero válido estadísticamente $\left(\mathrm{X}^{2}=3.89 ; \mathrm{p}<0.05\right)$ - que sus compañeros de niveles inferiores. La religión, por otro lado, parece ser una condición muy importante e insistente a la hora de determinar ciertas conductas relacionadas con la violencia: los jóvenes que no pertenecen a alguna confesión religiosa reportaron el mayor porcentaje de portación de cualquier tipo de armas en el transcurso de un mes. Finalmente, el estudio encontró que los jóvenes que tienen responsabilidades laborales, esto es, que tienen empleo, son los que admitieron la portación de amas con mayor frecuencia.

Por el otro lado, las variables que no aparecieron asociadas a la portación de amas dentro de los centros estudiantiles son la edad (clasificada en tres grupos) y el tipo de institución (aglutinado como público y privado). Los porcentajes exhibidos en el Cuadro 2 revelan, no obstante, que en el caso de la edad, parece haber un incremento en el acarreo de cualquier tipo de arma dentro de la escuela en la medida en que los jóvenes poseen más años. La verdad es que, por un leve margen, esta tendencia no está suficientemente respaldada de forma estadística $\left(X^{2}=5.82 ; p>0.05\right) y$, por tanto, esas diferencias en los porcentajes podrían deberse al azar y no a un efecto propio de la edad como tal. En el caso del tipo de institución hay que decir que ciertamente los datos no reflejan diferencia alguna en los comportamientos de acarreo de armas dentro de la escuela en virtud de la administración pública o privada de la misma; esto no signifíca, sin embargo, 


\section{Cuadro 2}

Porcentaje de estudiantes que han portado algún tipo de arma en el centro educativo en el lapso de un mes según variables

\begin{tabular}{lrr}
\hline & \multicolumn{2}{c}{ Portación de armas } \\
\cline { 2 - 3 } Variables & No & Sí \\
\hline Sexo & & \\
Masculino & 76.1 & 23.9 \\
Femenino & 90.7 & 9.3 \\
& & \\
Edad & & \\
De 13 a 14 años & 85.2 & 14.8 \\
De 15 a 17 años & 83.9 & 16.1 \\
18 años y más & 77.5 & 22.5 \\
& & \\
Nivel educativo & & \\
Básica & 85.6 & 14.4 \\
Bachillerato & 80.7 & 19.3 \\
& & \\
Religión & & \\
Ninguna & 74.1 & 25.9 \\
Católica & 84.2 & 15.8 \\
Evangélica & 85.8 & 14.2 \\
Otra & 88.9 & 11.1 \\
Tipo de institución & & \\
Pública & & \\
Privada & 82.2 & 17.8 \\
Condición laboral & 82.8 & 17.2 \\
Sí trabaja & & \\
No trabaja & & \\
\hline & 70.8 & 29.2 \\
\hline & 83.8 & 16.2 \\
\hline
\end{tabular}

que no existan diferencias sustantivas en el comportamiento de los estudiantes en función del instituto al cual pertenecen. Los análisis ulteriores de los datos de portación de armas, según los centros educativos, reveló que no en todos las instituciones se presenta la misma afición por la armas de parte de los estudiantes. Por ejemplo, en un centro educativo laico de administración privada y con una numerosa población estudiantil, el porcentaje de estudiantes armados llegó hasta el 49 por ciento; en tanto que en el instituto público más grande del país el 
porcentaje de acarreo de armas no superó al 15 por ciento; es más, algunas instituciones privadas de índole religiosa mostraron niveles de acarreo de armas mayores que otras de tipo laico o privado. En tal sentido, las diferencias entre las instiluciones educativas existen, sólo que no responden a una simple clasificación de instituto público y privado; probablemente el factor más importante en estos casos sería el régimen de disciplina y control que impera en cada una de las instituciones.

\subsection{La asociación de las conductas agresivas y la victimización con la porta- ción de armas dentro de la escuela}

Uno de los hallazgos más importantes del estudio de las conductas violentas de los adolescentes educandos del Area Metropolitana de San Salvador es el que se refiere a la asociación que existe $\longrightarrow$ parece existir- entre las conduclas relacionadas con la violencia. Más concretamente, los datos sugieren que las conductas de agresión dentro de la escuela hacia los otros compañeros, tanto como los eventos de victimización sufridos en el interior de la misma, estarían vinculados con el comportamiento de acarrear armas dentro de la escuela o centro educativo. En otras palabras y tal como lo muestra la Gráfica 2, los jóvenes que trajeron armas al interior de la institución tuvieron significativamente un promedio mayor de comportamientos agresivos y sufrieron más frecuentemente por la agresión de otros compañeros?. Y no sólo eso, la misma comparación se realizó con las conductas que "resultaron en heridas como para ser tratado por un médico o enfermera" y los datos revelaron una diferencia sustancial entre los que portaron armas y los que no $(F=50.4, p<0.0001)$.

\section{Grálica 2}

Indices de agresión y victimización en educativo según portación de arma

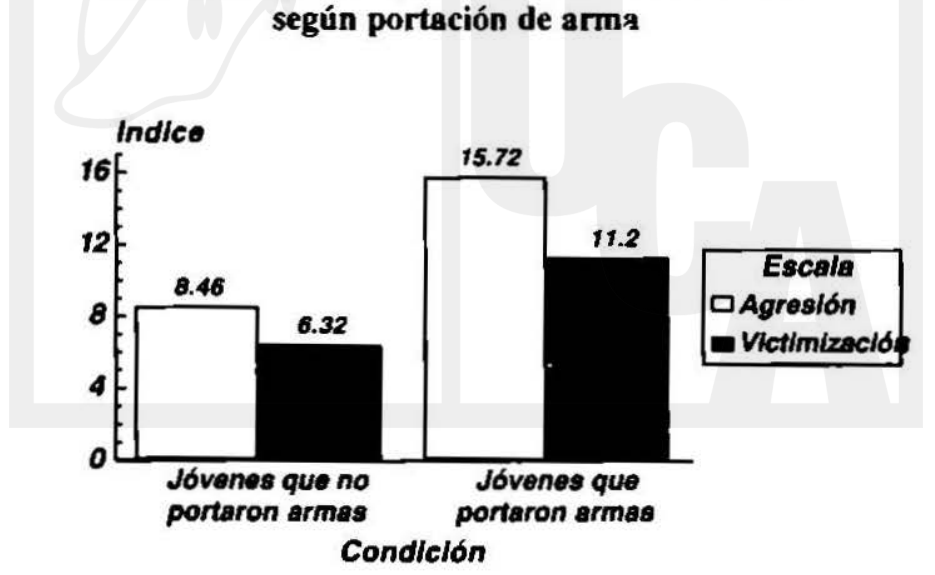


Lo anterior significa $-y$ reitera la idea que ha venido deslizándose a través de los datos anteriores- de que las conductas relacionadas con la agresión, y en sus formas más severas con la violencia, no son aisladas. Es decir, cuando los datos reportan a jóvenes que agreden a sus compañeros de clase y de institución con cierta frecuencia, están también recogiendo índices que muestran un elevado nivel de victimización por el mismo tipo de conductas de sus compañeros, lo que indica que las conductas de agresión se dan en dinámicas de reciprocidad en las que los jóvenes suelen someterse con más o menos frecuencia; pero además los datos indican que esta gente sería la más propensa - no necesariamente lo va a ser siempre - a conducirse armado de cualquier instrumento dentro del recinto escolar. Lo que dicen estos datos es que un joven que suele involucrarse en hechos de violencia con más frecuencia, suele ser víctima con más frecuencia y suele ser más peligroso — desde su comportamiento de acarreo de armas-con más frecuencia.

De hecho, un ejercicio de regresión lineal, usando la escala de agresión como variable dependiente, reveló que la victimización y la portación de armas explican simultáneamente hasta un 36 por ciento $\left(r^{2}=0.3607\right)$ de la variabilidad de las conductas de agresión entre los jóvenes consultados.

\section{Conclusiones y reflexiones}

Los resultados del estudio sobre violencia en los centros educativos de educación media revelan varias cosas que son importantes para iniciar un acercamiento al fenómeno de la violencia asociada con los jóvenes estudiantes.

En primer lugar, los datos expuestos en esta breve presentación con fines descriptivos muestran la frecuencia con que los jóvenes se comportan agresivamente dentro de sus centros escolares en el Area Metropolitana de San Salvador. De hecho, uno de cada cinco estudiantes de educación básica o media han protagonizado una pelea en el recinlo escolar en el lapso del último mes. Esta conducla de agresión intra-escolar parece no estar aislada de otros comportamientos de violencia fuera del entomo escolar y que pueden acarrear perjuicios para los mismos jóvenes; es más, los datos indican que existe una asociación significativa entre las conductas de molestar dentro del colegio y la de verse involucrado en actos de violencia que terminan en lesiones. Al mismo tiempo los datos sugieren que el problema de las conductas de agresión es un problema que se concentra mucho más en los adolescentes del sexo masculino y que poseen menos años de edad. Práclicamente un púber del sexo masculino tiene tres veces más conductas agresivas que una adolescente escolarizada de 19 años de edad, sobre todo si no posee una confesión religiosa. Efectivamente, la religión apareció como una variable fuertemente asociada a las conductas de los jóvenes; los estudiantes menos agresivos son aquéllos que pertenecen a confesiones religiosas que suelen vivir más intensamente su fe, pero esto no quiere decir que en los inslitulos administrados 
por religiosos la conducta estudiantil sea más pacífica, no. La variable religión parece actuar mejor en términos individuales que institucionales.

$\mathrm{Y}$ es que, en segundo lugar, los datos no ofrecen evidencias de que otras variables, como el nivel educalivo, agrupado en cursos de tercer ciclo y de bachillerato, o como el lipo de centro educativo (público o privado), tengan alguna relación con la frecuencia de las conductas de agresión dentro de las instituciones. En el primer caso, el peso mayor parece descansar en la edad de los jóvenes que en el nivel educativo en sí mismo; mientras que en el segundo caso, probablemente la amplia diversidad en los centros privados - frente a la mayor homogeneidad de las instituciones públicas- haga imposible encontrar un efecto determinante según el lipo de institución. Esto no quiere decir que no hayan diferencias importantes en la prevalencia de las conductas agresivas y, por ende, en la proclividad hacia la violencia más severa en ése o en otros entomos. De hecho, las comparaciones en los índices por agresión por cada una de las 34 instituciones educativas utilizadas para la muestra revelan disimilitudes importantes que, incluso, se mantienen o acentúan en las otras variables de violencia esludiadas. Hay claramente institulos que reportan unos índices de agresión en sus estudiantes muy elevados frente a otros que apenas sí muestran tales comportamientos entre sus estudiantes; pública e institución privada no es la mejor para indicar esas diferencias, y muestra que el problema de la violencia de los centros educativos no eslaría asociada al tipo de administración de la misma. En este caso, es necesario seguir profundizando en el análisis de los datos reclasificando las instiluciones privadas y públicas, según otras variables (como tamaño de la institución, recursos, etc.), para poder precisar mejor el efecto de los entomos institucionales en la disciplina escolar $y$, por ende, en la conducta agresiva de los escolares.

En tercer lugar, la investigación encontró que los jóvenes más frecuentemente victimizados, o que sufren por comportamientos de agresión por otros estudiantes, son usualmente los que reportan una conducta también agresiva. En tal sentido, los adolescentes hombres y los que no poseen religión alguna son los que enfrentan con más frecuencia las conductas de agresión de sus compañeros. Esto no quiere decir que, por ejemplo, las mujeres o los más jóvenes no sean agredidos dentro de los entornos escolares; significa más bien que los estudiantes con aquéllas condiciones hacen frente a unos niveles de agresión por otros mayor que cualquier otro grupo.

En cuarto lugar, el estudio en cuestión anoja datos que muestran que de cada cien estudiantes que asisten a clases en sus centros educativos, al menos diecisiete se encuentran armados con cualquier instrumento. Las armas introducidas más frecuentemente a los centros escolares son las ammas blancas y los garrotes y palos; sin embargo, el porcentaje de estudiantes que admitió haber acarreado armas de fuego y explosivos no es desdeñable, considerando que de cada cien 
estudiantes, alrededor de cinco podrían estar armados con estos tipos de insiru. mentos. En este caso, los datos reiteran a los jóvenes del sexo masculino y a los no religiosos como las personas que suelen introducir ammas con más frecuencia; pero además revelan otra condición, los que tienen empleo. Por el contrario, la pertenencia a un tipo de institución, sea ésta pública y privada, no parece hacer diferencias en tomo a estas conduclas; pero sí lo hace el pertenecer a algunas instituciones concretas, donde probablemente la tolerancia hacia ese tipo de conductas sea mayor.

Pero, quizás, y en quinto lugar, uno de los hallazgos más importantes entre los resultados expuestos en esta breve comunicación es el que muestra que la porlación de armas está relacionada con esas conductas de agresión y victimización dentro de la escuela, y con la participación de hechos de violencia en cualquier entomo. Esto significa que los jovenes que portan amas poseen una inclinación más fuerte por las conductas agresivas que aquéllos que no las llevan y que, por tanto, son más propensos a usarlas en alguna circunstancia que el resto de jóvenes. Los resultados anteriores permiten pensar que los jóvenes que acuden a sus centros educativos portando armas de cualquier tipo no lo hacen por un simple comportamiento de alarde, como alguna gente argumenta para restarle importancia a este tipo de conductas, sino porque suelen utilizar las conductas de violencia y agresión como una forma de relacionarse con los demás.

Finalmente hay que decir que tales datos llaman a reflexión sobre el problema de la violencia juvenil en el país. Por una parte, el problema de la violencia juvenil en las calles viene de los enfrentamientos y riñas protagonizadas por las maras estudiantiles; por otro lado, la violencia juvenil está vinculada a las maras callejeras, cuyos integrantes, a su vez han sido parte de las maras estudiantiles (Cruz y Portillo, 1998). En tal sentido, la violencia juvenil de las calles salvadoreñas tiene su vinculación con lo que los jóvenes hacen y aprenden en los entomos escolares. Los datos de esta investigación han subrayado esa ligadura y sugieren que no es posible entender los comportamientos violentos de los jóvenes sin acudir a sus comportamientos dentro de las escuelas y a los entomos relacionales creados dentro de las mismas; como tampoco es posible aproximarse al fenómeno sin comprender que hay ciertas condiciones personales que facilitan el acercamiento del joven a la violencia y que, por tanto, pueden considerarse factores de riesgo, como el pertenecer al sexo masculino o el no profesar alguna religión.

Aunque la investigación no pudo encontrar la mejor forma de clasificar a las instituciones para relacionarla con los comportamientos violentos de sus estudiantes, es claro que algunas instiluciones permiten y toleran más las relaciones agresivas de sus estudiantes en el interior, al tiempo que toleran comportamientos que ponen en peligro la vida de otros al introducir armas y explosivos a una escuela. Este tipo de jóvenes, con aquéllas características, son los que en última instancia están más propensos a sumarse al fenómeno social de la violencia. 


\section{Bibliografía}

Cruz, Josć Migucl, La victimización por violencia urbana: niveles y faciores asociados en ciudades iberoamericanas, (Borrador mimcografiado), 1998.

Cruz, José Miguel, "Los factores posibilidades de la violencia y las expresiones de la violencia en los noventa", Estudios Centroamericanos (ECA), LIl, 588, San Salvador: UCA, 1997, pp. 977-992.

Cruz, José Miguel y Gonzálcz, Luis Armando, "La magnitud de la violencia en El Salvador", Estudios Centroamericanos (ECA), LII, 588, San Salvador: UCA, 1997, pp. 953-966.

Cruz, José Migucl y Portillo, Nclson, Solidaridad y violencia en las pandillas del gran San Salvador. Más allá de la vida loca, San Salvador: UCA Editores, 1998.

Iglesias, Enrique, "Los desafios de la criminalidad urbana", Documento especial, Proceso, 17, 753, San Salvador: UCA, 1997, pp. 13-16.

Instituto Universitario de Opinión Pública, "Sondeo sobre violencia en los centros educalivos del Área de San Salvador", Serie de informes 59, San Salvador. IUDOP-UCA, 1996.

Levine, Felice J. y Rosich, Katherinc J., Social causes of violence: Crafting a science agenda, Washington D.C.: American Sociological Asociation, 1996.

Lockwood, David, "Violence among middle school and high school students: analysis and implications for preventions", National Institute of Justice, Reaserch brief, Washington D.C., Office of Justice Programs, 1997.

Martínez, Mario, "Herido en pleito de «<maras»»", El Diario de Hoy, 1 de agosto de 1998, p. 12.

National Research Council, Understanding and preventing violence, Washinglon D.C.: Nalional Academy Press, 1993.

Norusis, Marija J., The SPSS guide for data analysis (2da. edición), Chicago: SPSS Inc., 1992.

Office of Juvenile Justice and Delincuency Prevention, Gang supression and intervention: problem and response. Research summary, Washington D.C.: Office of Justice Programs, 1994.

Orpinas, Pamela, Comparison of student and community violence in the U.S. and El Salvador, (Borrador mimeografiado), 1997.

Orpinas, Pamcla y Frankowsk, Ralph, La Escala de agresión: una medida autoaplicada de la conducta agresiva en esfudiantes de educación media (Borrador mimeografiado), 1996.

Smul, Marcela y Miranda, Jenny, El fenómeno de las pandillas en El Salvador, San Salvador: FLACSO-UNICEF, 1997.

Tolan, Patrick H., "Implications of age of onset for delincuency risk", Journal of Abnormal Child Psychology, 15 (1), 1987, pp. 47-65.

Urías, Tania, "Guerra estudiantil. De los cuadernos a las piedras", La Prensa Gráfica, 12 de septiembre de 1997, p. 43.

U.S. Department of Justice, Criminal victimization in the United Slates, 1994. A national crime victimization survey report, Washington, D.C.: Bureau of Justice Statistics, 1997.

Yunes, Joao, "Mortalidad por causas violentas en la región de las Américas", Boletín de la Oficina Sanitaria Panamericana, 114, 4, 1993, pp. 302-314.

Yunes, Joao Y Rajs, D., "Tendencia de la mortalidade por causes violentas en la poblacion general e entre los adolescenles y jóvenes de la regiao de las Americas", Cadernos de Saúde Pública, 10 (1), 1994, pp. 88-125. 


\section{Notes}

1. Director del Instituto Universitario de Opinión Pública de la Universidad Centroamericana "José Simeón Cañas" (UCA).

2. Parte de este artículo se basa en una ponencia presentada en el ler Congreso Nacional de Psicología "Lic. Francisco Armando Torres", el 5 de diciembre de 1997.

3. Traducción literal del aulor. La expresión en inglés es "onset for delincuency risk".

4. La pregunta rezaba de la siguiente forma: En los últimos 30 dias, ¿cuántas veces peleaste (pelear a golpes, tirar el pelo, morder) en la escuela?

5. En los últimos 30 dias, ¿cuantas veces te enfrentaste a golpes con otra(s) persona(s), y resultaste herido(a), como para ser iralado(a) por un médico o enfermera?

6. Esto según una prucba de Scheffé para comparaciones múltiples.

7. Un análisis de varianza entre las conductas de agresión y la portación de armas arrojó una $\mathbf{F}=89.2$, con una $p<0.0001$. En este caso, la Ela cuadrada reveló que el 8 por ciento de la varianza en los índice de agresión se puede atribuir a la condición de portación de armas. Por otro lado, el análisis de varianza realizado entre la victimización y la portación de armas arrojó una $F=65.9$, con una $p<0.0001$. Eta cuadrada $=0.062$.

8. De hecho, los datos señalaron a las instituciones que, por lo general, sus estudiantes se ven involucrados en hechos de violencia o en riña callejeras; sin embargo, en esta presentación se decidió no presentar los nombres de las mismas porque los investigadores adquirieron el compromiso con todas las instiluciones incluidas $\mathrm{en} \mathrm{la} \mathrm{muestra}$ de no señalar los nombres de las mismas en los resultados. 